\title{
Decreased cerebral blood flow in the limbic and prefrontal cortex using SPECT imaging in a cohort of completed suicides
}

\author{
K Willeumier, DV Taylor and DG Amen
}

Suicide has a high comorbidity with impulsivity and depression, and finding imaging biomarkers indicative of patients at high risk for suicidal behavior is invaluable to the clinician. Using single-photon emission computed tomography (SPECT) imaging, we have previously reported regional cerebral blood flow (rCBF) decreases in the medial prefrontal cortex, ventral tegmental area and subgenual cingulate cortex (Brodmann area 25 (BA 25)), a region found to be hypoperfused with treatment-resistant depression. From 2007 to 2010, we have extended our analysis to include nine additional completed suicides. In all, 27 healthy, age- and gender-matched subjects from a previously acquired healthy brain study served as controls to our 21 completed suicides. All 21 suicides had been previously diagnosed with depression according to Diagnostic and Statistical Manual of Mental Disorder-IV criterion. Voxel-by-voxel analyses were performed using statistical parametric mapping to compare the differences in technetium-99m hexamethylpropylene amine oxime brain uptake between the groups. Factor analysis of the data identified the top 10 regions of hypoperfusion in the suicidal group, including the bilateral superior frontal lobes, the right precuneus, the rolandic operculum, postcentral gyrus, left caudate and insular cortex. We also demonstrate more focal decreases in rCBF in the subgenual cingulate cortex (BA 25) in 18 subjects, supporting our previous hypothesis that hypoperfusion of BA 25 may be a risk factor for suicide in depressed patients. This work suggests that SPECT might be useful in predicting risk for suicide completion in subjects with depression or treatment-resistant depression. Further investigation of this work is necessary to better understand the predictive value of this finding.

Translational Psychiatry (2011) 1, e28; doi:10.1038/tp.2011.28; published online 9 August 2011

\section{Introduction}

The World Health Organization estimates that 1000000 people die yearly from suicide. ${ }^{1}$ Although suicide is growing at an alarming rate, it is a poorly understood psychiatric disorder and is currently ranked among the top 10 leading causes of death in the United States, ${ }^{2}$ with 16 suicides committed per every 100000 individuals worldwide. ${ }^{1}$ Analysis of data from the US National Comorbidity Survey-Replication, the first nationally representative mental health survey in the United States, revealed that $80 \%$ of suicide attempts were due to mental disorders, primarily related to anxiety, mood, impulse control and substance abuse. ${ }^{3}$ Of the mental disorders, major depressive disorder is considered one of the top four psychiatric risk factors associated with an increased predisposition towards suicidal ideation and attempted suicide. ${ }^{4}$ The use of anatomical and functional imaging studies to expand our understanding of the neurobiology underlying suicide is clearly needed.

The etiology of suicide is complex, and most likely it is the subtle neuroanatomic changes that result in the social and emotional problems observed in these patients. As suicide has many comorbid risk factors, determining causality requires understanding the extent to which comorbidity effects brain structure and function. Bipolar disease is one such comorbidity, and several neuroanatomic imaging studies suggest that this is a disorder that results in decreased gray matter volume in the subgenual prefrontal cortex (Brodmann area 25 (BA 25)). ${ }^{5-7}$ The subgenual prefrontal cortex is important in the regulation of mood and emotions, and bilateral decreases in brain volume have been observed in individuals with major depressive disorder. ${ }^{8}$ We have previously reported a decrease in rCBF in the subgenual prefrontal cortex in a small cohort of completed suicides. ${ }^{9}$ Interestingly, in patients with treatment-resistant depression, single-photon emission computed tomography (SPECT) imaging has been used to show increased rCBF in the subgenual cingulate gyrus in patients who were positive responders to citalopram. ${ }^{10}$ Taken together, these results suggest that decreased activity of the subgenual cingulate gyrus in those with depression or mood disorder may be predisposed to suicidal tendencies.

The aim of this study was to retrospectively analyze a cohort of psychiatric patients who have committed suicide and compare the changes in $\mathrm{rCBF}$ to that of a previously acquired healthy brain cohort. Our prior data on 12 completed suicides revealed a unique finding of hypoperfusion in the subgenual cingulate cortex (BA 25). Furthermore, we analyzed this cohort against a group of depressed, non-suicidal subjects with similar 
clinical profiles. Overall, we aim to further characterize the underlying rCBF deficits in this cohort of completed suicides who exhibit depression as a comorbid factor.

\section{Methods}

Using a previously acquired database of over 64000 SPECT scans from patients seen across four clinics in the United States, we retrospectively pulled the data of 21 completed suicides, which occurred from 1992 to 2010 . Within this time frame, 38000 patients had been evaluated at one of the clinics, of which $74 \%$ were diagnosed as having a mood disorder or depression. All patients in the suicide group had been previously evaluated by a board-eligible or -certified psychiatrist for depression. They each underwent a detailed clinical history, mental status examination and Beck Depression Inventory (BDI). Table 1 outlines the patient demographics and psychiatric history and Table 2 gives a profile of the medication history for each patient at the time of the visit. All of the subjects fit the clinical criteria for depression based on the BDI, Diagnostic and Statistical Manual of Mental Disorder-IV (DSM-IV) or had been given a diagnosis of mood

Table 1 Suicide cohort demographics and psychiatric diagnoses

\begin{tabular}{|c|c|c|c|c|c|c|c|c|c|c|c|c|c|c|}
\hline $\begin{array}{l}\text { Age } \\
\text { (years) }\end{array}$ & Gender & $B D I$ & $\begin{array}{l}\text { Depre- } \\
\text { ssed }\end{array}$ & $\begin{array}{c}\text { Mood } \\
\text { disorder }\end{array}$ & Bipolar & $\begin{array}{c}\text { Suicidal } \\
\text { ideations }\end{array}$ & $\begin{array}{l}\text { Suicide } \\
\text { attempt }\end{array}$ & $A D H D$ & PTSD & Anxiety & $\begin{array}{c}\text { Drug } \\
\text { use }\end{array}$ & $\begin{array}{c}\text { Head } \\
\text { trauma }\end{array}$ & $\begin{array}{l}\text { Sexual } \\
\text { abuse }\end{array}$ & $\begin{array}{l}\text { Months } \\
\text { to death }\end{array}$ \\
\hline 41 & $M$ & NA & $x$ & & & & & & $x$ & & & & & 14 \\
\hline 51 & $M$ & 19 & & $x$ & & & $x$ & & & & & & & 3 \\
\hline 41 & $\mathrm{~F}$ & 36 & $x$ & & & & & $x$ & & $x$ & & & & 89 \\
\hline 26 & $M$ & 5 & & $x$ & & & $x$ & & & & $x$ & $x$ & & 1 \\
\hline 64 & $M$ & 27 & $x$ & & & & & & & & & & & \\
\hline 42 & $\mathrm{~F}$ & 40 & & $x$ & $x$ & & & $x$ & & $x$ & & & & 12 \\
\hline 50 & $M$ & NA & $x$ & & & & & $x$ & & & & $x$ & & 9 \\
\hline 21 & $M$ & 19 & & $x$ & & $x$ & $x$ & $x$ & & & $x$ & & & \\
\hline 21 & $\mathrm{M}$ & 34 & $x$ & & & $\hat{x}$ & $\hat{x}$ & & & & $\hat{x}$ & $x$ & $x$ & 14 \\
\hline 36 & $\mathrm{~F}$ & NA & $\hat{x}$ & & & $\hat{x}$ & $\hat{x}$ & & & $x$ & & $\hat{x}$ & & 6 \\
\hline 15 & $M$ & 99 & $x$ & & & & $x$ & $x$ & & $x$ & $x$ & $\hat{x}$ & & \\
\hline 39 & $\mathrm{M}$ & 46 & & & $x$ & $x$ & $\hat{x}$ & $\hat{x}$ & & $\hat{x}$ & $\hat{x}$ & $\hat{x}$ & $x$ & \\
\hline 63 & $\mathrm{M}$ & 38 & $x$ & & $\hat{x}$ & $\hat{x}$ & $\hat{x}$ & & & $\hat{x}$ & & $\hat{x}$ & & 37 \\
\hline 43 & $M$ & 24 & $x$ & & & $x$ & $x$ & $x$ & & $x$ & $x$ & $x$ & $x$ & 15 \\
\hline 33 & $M$ & 31 & & $x$ & & & $x$ & $x$ & $x$ & & $x$ & $x$ & & 2 \\
\hline 20 & $M$ & 11 & $x$ & & & & $x$ & $x$ & & & & & & 9 \\
\hline 45 & $\mathrm{~F}$ & 32 & $x$ & & & $x$ & $x$ & $x$ & & & & & & 7 \\
\hline 19 & $M$ & 16 & & $x$ & & & $x$ & $x$ & & & $x$ & $x$ & & \\
\hline 34 & $M$ & NA & $x$ & & & $x$ & $x$ & $x$ & & & $x$ & $x$ & $x$ & 45 \\
\hline 28 & $\mathrm{~F}$ & 34 & & $x$ & & & $x$ & $x$ & & & $x$ & & & 20 \\
\hline 23 & $M$ & NA & & $\hat{x}$ & & $x$ & $\hat{x}$ & & $x$ & $x$ & $\hat{x}$ & $x$ & & 7 \\
\hline
\end{tabular}

Abbreviations: ADHD, attention deficit hyperactivity disorder; BDI, Beck Depression Inventory; DSM-IV, Diagnostic and Statistical Manual of Mental Disorder-IV; NA, not applicable; PTSD, post-traumatic stress disorder; SPECT, single-photon emission computed tomography.

In all, 21 subjects ranging in age from 15 to 64 years previously underwent a psychiatric evaluation and brain SPECT imaging. A BDI score of 25 or greater results in the diagnosis of depression as defined by the DSM-IV criterion. An ' $x$ ' in the category of depression, mood disorder or bipolar was given based on a diagnostic evaluation from a treating psychiatrist. The mean time from clinic visit to suicide was $18 \pm 22$ months. The exact time of death data was unavailable for five of the subjects listed.

Table 2 Suicide cohort medication history

\begin{tabular}{|c|c|c|c|c|c|c|}
\hline Patient & Antidepressants & $\begin{array}{c}\text { Antianxiety } \\
\text { medications }\end{array}$ & $\begin{array}{c}\text { Mood } \\
\text { stabilizers }\end{array}$ & $\begin{array}{c}A D H D \\
\text { medications }\end{array}$ & Antipsychotic & Sleep aid \\
\hline 1 & & & & & $x$ & \\
\hline $\begin{array}{l}2 \\
3^{a} \\
4^{a}\end{array}$ & $x$ & & & $x$ & & \\
\hline 5 & $x$ & & $x$ & & $x$ & \\
\hline 6 & $x$ & & & & $x$ & \\
\hline 7 & $x$ & & $x$ & $x$ & $x$ & \\
\hline 8 & $x$ & $x$ & $x$ & & $x$ & \\
\hline 9 & $x$ & & $x$ & & $x$ & \\
\hline 10 & $x$ & & & $x$ & & \\
\hline 11 & $x$ & $x$ & $x$ & & & \\
\hline 12 & $x$ & & $x$ & $x$ & & $x$ \\
\hline 13 & $x$ & & $x$ & & & \\
\hline 14 & $x$ & & $x$ & & $x$ & $x$ \\
\hline 15 & $\hat{x}$ & & $x$ & & $x$ & $x$ \\
\hline 16 & $x$ & & & & $x$ & $x$ \\
\hline $\begin{array}{l}17 \\
18^{a}\end{array}$ & $x$ & & & & & \\
\hline 19 & $x$ & & $x$ & $x$ & & $x$ \\
\hline 20 & & & & & $x$ & \\
\hline 21 & & & & & $x$ & \\
\hline
\end{tabular}

Abbreviation: ADHD, attention deficit hyperactivity disorder.

All 21 subjects were off medications for the appropriate washout period before being scanned. The medication history of each patient listed is at the time of visit. ${ }^{a}$ Medication history was not available. 
disorder. Within this group, 16 patients had at least one prior suicide attempt, and nine had expressed suicidal ideations. In addition, 13 patients had been diagnosed with attention deficit hyperactivity disorder (ADHD) as measured by DSM-IV criteria. A total of 12 patients had experienced prior head trauma and 11 had problems with substance abuse. In all, 27 subjects previously acquired under an institutional review board-approved healthy brain study were selected who were within 5 years of age and the same gender as at least one member of the suicide cohort. Two participants in our suicide group were not age matched within 5 years as we did not have elderly males aged 63 and 64 years in our healthy brain subjects (HBSs). The mean age of the suicide cohort was $36 \pm 14.0$ years (15-64 years), whereas the mean age of the healthy brain cohort was $35 \pm 9.6$ years (20-52 years).

We next compared our group of 21 completed suicides to a control group of 36 non-suicidal depressed patients. Depressed controls were selected by using a search algorithm that matched each subject with someone of the same gender who was within 10 years of age, without suicide ideation or history of suicide attempt and whose diagnostic profile matched the criteria of having depression, anxiety disorder, bipolar disorder and head trauma. Two passes of the search algorithm were run to arbitrarily select two matches for each suicide subject. Only one match was found for each subject on the first pass and on the second pass matches were found for 15 subjects giving a set of 36 non-suicidal depressed controls with an average age of $36 \pm 15$ years (15-66 years).

SPECT scans. Each subject underwent high-resolution brain SPECT imaging to measure regional cerebral blood flow (rCBF) as part of their evaluation. Each subject received an age/weight-appropriate dose of technetium-99m hexamethylpropylene amine oxime intravenously. Subjects were injected in normal lighting while they performed the Conner's Continuous Performance Test II. The radiopharmaceutical was injected $3 \mathrm{~min}$ after starting the 15-min test. All subjects completed the task. Subjects were then scanned 30 min later using a high-resolution Picker Prism 3000 tripleheaded gamma camera with fan beam collimators, acquiring data in $128 \times 128$ matrices, yielding 120 images per scan, with each image separated by $3^{\circ}$ spanning $360^{\circ}$.

SPECT data were processed and attenuation correction performed using general linear (Chang) methods. All images were reconstructed and resliced using an oblique reformatting program, according to anterior-posterior commissure line, so that the final images were similarly aligned for analysis.

Image analysis. Differences in hexamethylpropylene amine oxime uptake were analyzed using the SPM8 software (Wellcome Department of Cognitive Neurology, London, UK) implemented on the Matlab platform (MathWorks Inc., Sherborn, MA, USA). Statistical parametric maps (SPMs) are spatially extended statistical processes that are constructed to test hypotheses about regionally specific effects in neuroimaging data. SPM combines the general linear model and the theory of Gaussian random fields to make statistical inferences about regional effects. ${ }^{11}$ The images were spatially normalized using a 12-parameter affine transformation, followed by nonlinear deformations ${ }^{12}$ to minimize the residual sum of squares between each scan and a reference or template image conforming to the standard space defined by the Montreal Neurological Institute template. The original image matrix obtained at $128 \times 128 \times 29$ with voxel sizes of $2.16 \times 2.16 \times 6.48 \mathrm{~mm}^{3}$ was transformed and resliced to a $79 \times 95 \times 68$ matrix with voxel sizes of $2 \times 2 \times 2 \mathrm{~mm}^{3}$ consistent with the Montreal Neurological Institute template. Images were smoothed using an $8 \mathrm{~mm}$ full-width at half-maximum isotropic Gaussian kernel.

Data analysis. We compared the SPECT scans from the suicide cohort versus the healthy brain cohort using SPM with a two-sample $t$-test using covariance to control for the effects of age and gender. Factorial modeling in SPM was used for regression of the binary variable having completed suicide against rCBF while using covariance to control for the effects of age and gender.

\section{Results}

We age and gender matched our suicide cohort of 21 subjects with 27 HBSs. Within the suicide cohort, all had met the clinical criteria of having depression as measured by the BDI and DSM-IV. Additional comorbidities within this group include attention deficit hyperactivity disorder, post-traumatic stress disorder, anxiety, substance abuse and head trauma (see Table 1). Over half of the subjects had dual diagnoses of depression with ADHD, substance abuse or head trauma. In total, 16 subjects report one prior suicide attempt, with nine having experienced incidence of suicidal ideations. Issues with post-traumatic stress disorder, physical violence and sexual abuse were also reported within this cohort, but the incidence was low. Within the HBS group, each had met the clinical criteria for a healthy brain subject, which included the absence of current medical illnesses, past brain trauma, family history of psychiatric illness, current or past drug or alcohol abuse and no current or past evidence of neurological, behavioral or psychiatric issues as measured by a detailed clinical history, Minnesota Multiphasic Personality Inventory and Structured Clinical Interview for Diagnosis for DSM-IV. The mean age for the suicide cohort was $36 \pm 14.0$ years (16 men, 5 women), whereas for the HBS cohort, it was $35 \pm 9.6$ years (17 men, 11 women). SPM analysis on the concentration brain SPECT imaging scans of the suicide group revealed diffuse regions of hypoperfusion across the brain at $P<1 \times 10^{-10}$ family-wise error (FWE) corrected as compared to the HBS group. Figure 1 depicts the threedimensional (3D) cortical surface views showing global brain SPECT decreases in rCBF in the suicide subjects.

To determine which deficits in rCBF were most predictive of being in the suicide group, we used multiple regression analysis within SPM and controlled for age and gender. The 10 most significant regions of decreased rCBF in terms of cluster size that were found to be most predictive of the suicide cohort are listed in Table 3. Factor analysis revealed the five most predictive areas of reduced blood flow in suicide patients include the bilateral superior frontal lobes, right precuneus, rolandic operculum, postcentral gyrus and left caudate-thalamus. The regional areas as identified through 


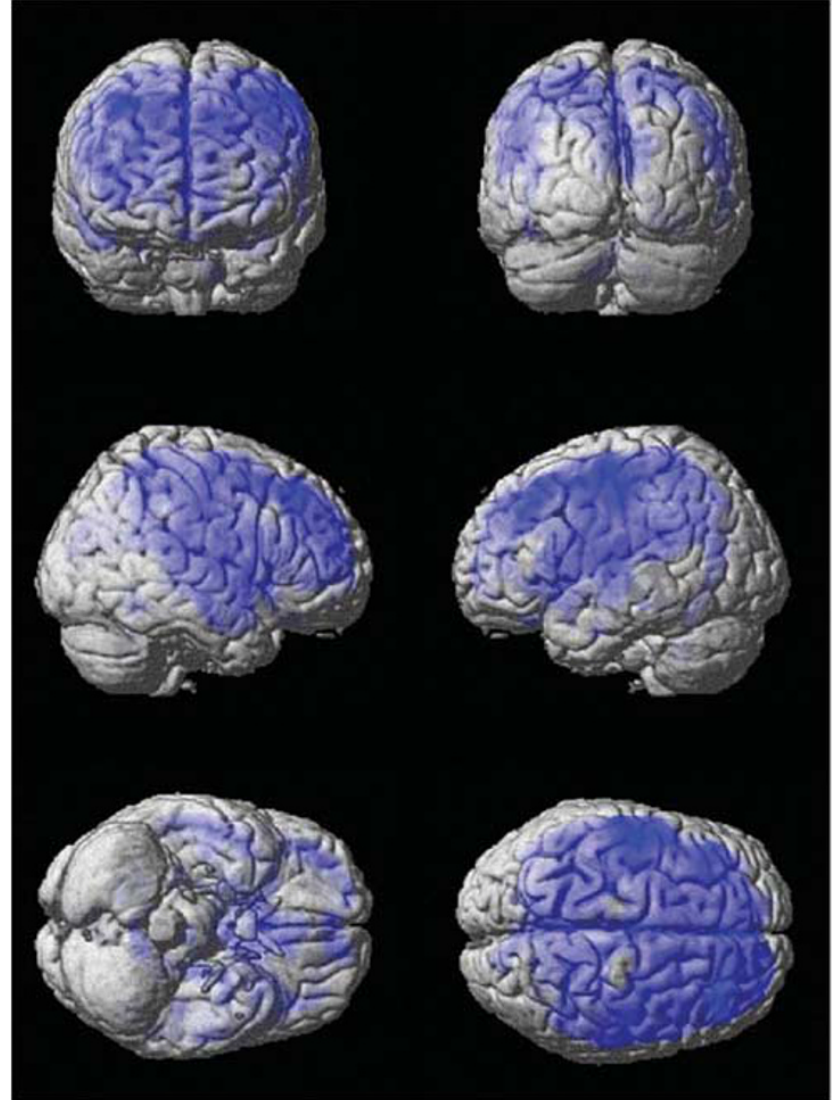

Figure 1 Three-dimensional (3D) surface images showing global brain singlephoton emission computed tomography (SPECT) decreases in suicide subjects versus healthy brain subjects. Cortical surface views show decreased regional cerebral blood flow in suicide subjects at $P<1 \times 10^{-10}$, family-wise error (FWE). Significant decreases are in blue. No increases were observed.

Table 3 Top 10 regions of decreased $\mathrm{rCBF}$ predictive of suicide relative to a healthy brain cohort at $P<0.05 \mathrm{FWE}$

\begin{tabular}{lccrrr}
\hline Brain area & Cluster size & $\boldsymbol{t}$ & \multicolumn{1}{c}{$\boldsymbol{x}$} & \multicolumn{1}{c}{$\boldsymbol{y}$} & $\boldsymbol{z}$ \\
\hline Precuneus & 520 & 4.84 & 18 & -48 & 16 \\
Right frontal lobe & 505 & 4.53 & 14 & 46 & 46 \\
Left frontal lobe & 373 & 4.35 & -16 & 48 & 42 \\
Rolandic operculum & 345 & 4.17 & 42 & -12 & 22 \\
Postcentral left & 262 & 4.12 & -48 & -12 & 58 \\
Caudate-thalamus & 317 & 4.11 & -12 & -16 & 22 \\
Insula & 260 & 4.1 & 32 & 18 & 12 \\
Caudate-putamen-R & 427 & 3.99 & 10 & 12 & 12 \\
Frontal inferior orbital & 90 & 3.96 & 28 & 36 & -4 \\
Insula-putamen & 64 & 3.91 & -26 & 20 & 12 \\
& & & & &
\end{tabular}

Abbreviation: rCBF, regional cerebral blood flow.

SPM analysis are as follows: Region 1 shows the most significant decrease in rCBF with an overall cluster size of 520 voxels and is located in the right precuneus (coordinates 18 , $-48,16 ; t=4.84)$. Region 2 encompasses the frontal lobes with a cluster size of 505 voxels in the right frontal superior medial cortex (coordinates 14, 46, 46; $t=4.53$ ) and 373 voxels in the left frontal superior cortex (coordinates -16 ,

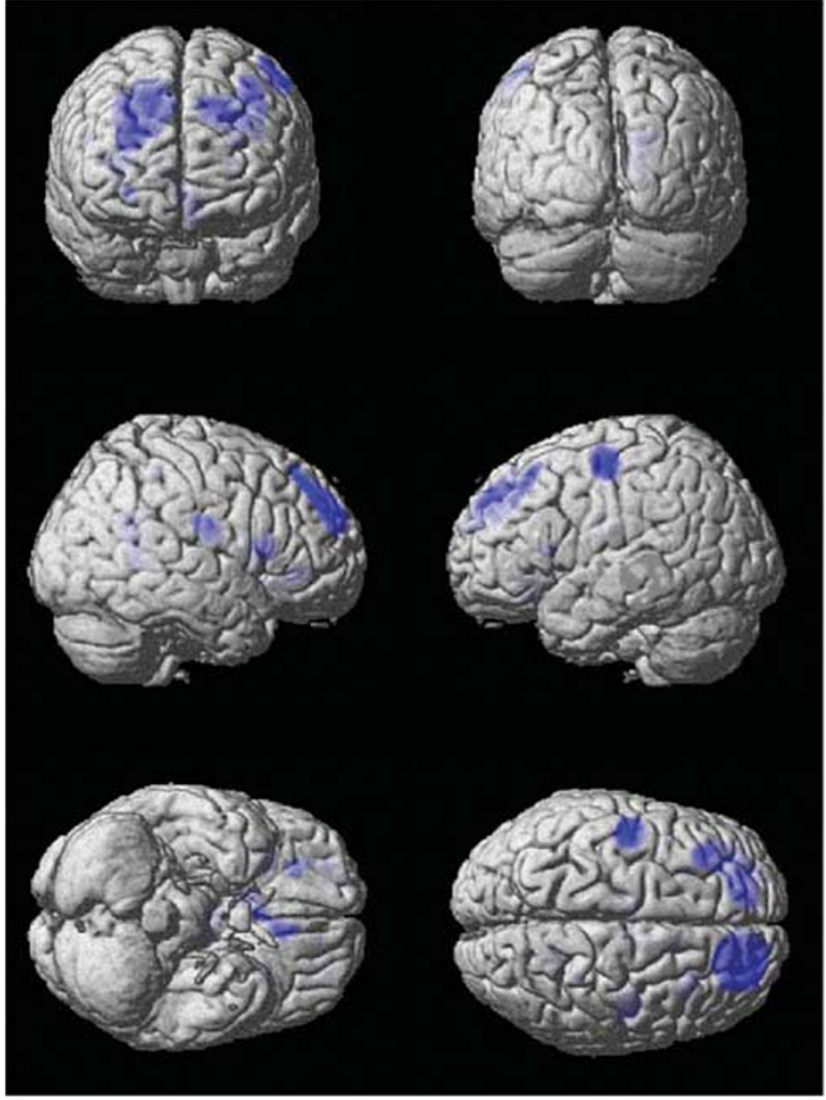

Figure 2 Three-dimensional (3D) surface images showing regional cerebral blood flow (rCBF) deficits predictive of suicide. Cortical surface views show areas of decreased $\mathrm{rCBF}$, which predict suicide at $P<0.05$, family-wise error (FWE) Significant decreases are in blue.

$48,42 ; t=4.35)$. Regions $3-5$ include the rolandic operculum (coordinates $42,-12,22 ; t=4.17$ ), left postcentral gyrus (coordinates $-48,-12,58 ; t=4.12$ ) and left caudate (coordinates $-12,-16,22 ; t=4.11$ ). Figure 2 shows the 3D cortical representation of these brain regions and Figure 3 illustrates these areas through transverse, coronal and sagittal slices $(P<0.05$, FWE).

A region-of-interest analysis was applied to BA 25. Individual analysis revealed that 18 subjects had decreased rCBF in BA 25 as compared with HBSs. Of these subjects, 13 were $1 \mathrm{~s} . \mathrm{d}$. below the mean from the HBS. Group analysis showed that the suicide cohort had a lower overall average rCBF in BA 25 as compared with our healthy brain cohort at $P=0.001$ and $t=3.45$ (suicide cohort: $509 \pm 200$; HBS: $668 \pm 115)$. No significant increases were observed.

To further differentiate the changes in rCBF in our suicide cohort from a group of depressed patients with a similar clinical profile who are non-suicidal, we performed an SPM analysis on concentration SPECT scans and found diffuse regions of hypoperfusion in $\mathrm{rCBF}$ throughout the brain of the suicide group at $P<1 \times 10^{-5}$ FWE. Figure 4 depicts the 3D cortical surface views showing global brain SPECT decreases. When comparing available BDI scores, the suicide cohort $(n=15)$ scored higher $(27.5 \pm 11.5)$ than the nonsuicidal cohort $(n=27)(15.0 \pm 7.0)\left(t_{(40,2)}=-4.36, P<0.01\right)$. 


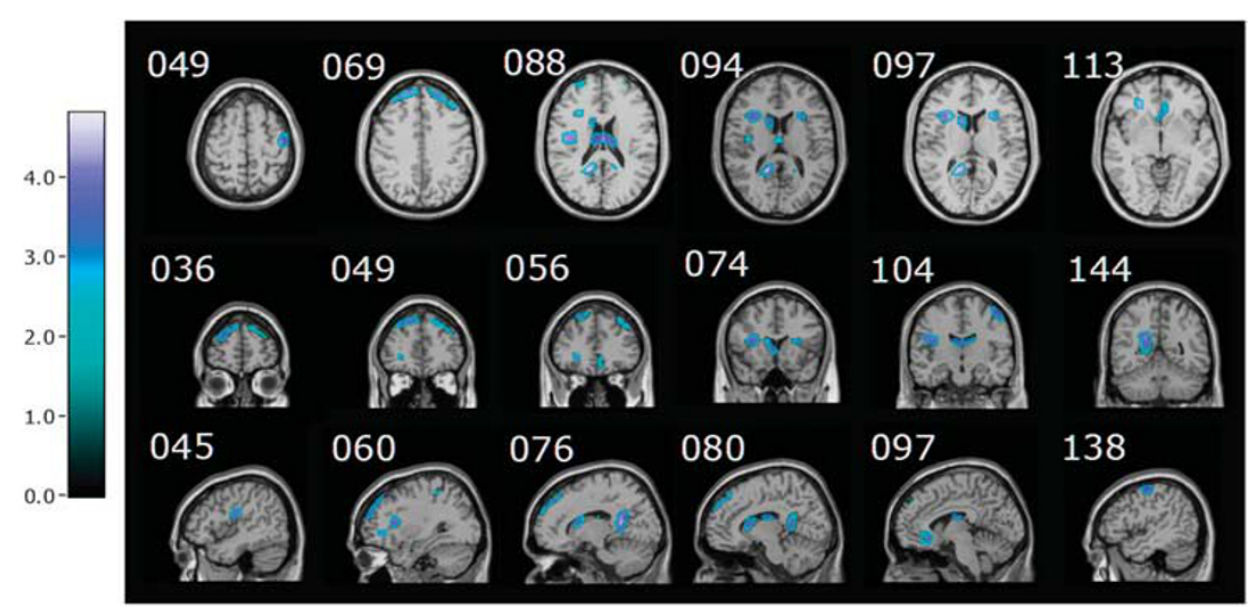

Figure 3 Image sections showing regional cerebral blood flow (rCBF) decreases predictive of completing suicide. Transverse, coronal and sagittal sections showing predictive areas of suicide: Transverse 49-postcentral left; 69-right and left frontal areas; 88-frontal inferior right, postcentral right, caudate right, thalamus right, precuneus right; 94-frontal inferior right, postcentral right, caudate right, thalamus right, precuneus right; 97-insular cortex right, caudate right, insular cortex left, precuneus right; 113-insular cortex right, frontal inferior orbital left (Brodmann area (BA) 25). Coronal 36-left and right frontal lobes; 49-frontal superior right, frontal superior left, insular cortex; 56 frontal superior right, frontal superior left, inferior frontal right, frontal inferior orbital left (BA25); 74-insular cortex right, caudate right, insular cortex left; 104-rolandic operculum right, thalamus right, postcentral left, 144-precuneus right. Sagittal 45-rolandic operculum right; 60-frontal cortex right, insular cortex right, frontal inferior cortex right (BA 25); 76-frontal cortex, caudate right, precuneus; 80-frontal cortex, caudate, thalamus right, precuneus; 97-frontal inferior orbital left (BA 25), thalamus; 138-postcentral left.

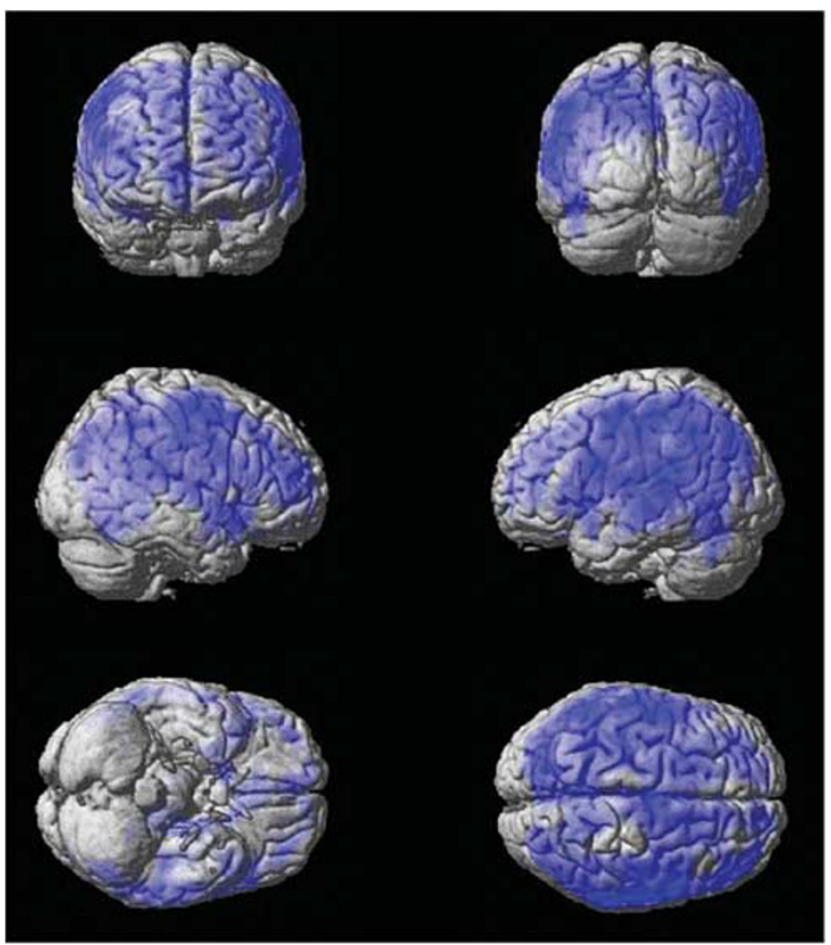

Figure 4 Three-dimensional (3D) surface images showing global brain singlephoton emission computed tomography (SPECT) decreases in suicide cohort versus depressed non-suicidal cohort. Cortical surface views show diffuse regions of decreased regional cerebral blood flow in suicide subjects at $P<1 \times 10^{-5}$, family-wise error (FWE) in comparison to non-suicidal subjects with matched diagnostic profiles. Significant decreases are in blue. No increases were observed.

Regression analysis across our healthy brain cohort, depressed non-suicidal cohort and suicide cohort showed that $\mathrm{rCBF}$ throughout the brain continued to decrease with severity of illness $\left(P<1 \times 10^{-10}\right)$.
A regression model demonstrating decreases in $\mathrm{rCBF}$ predictive of suicide using the two clinical populations revealed large, diffuse clusters throughout the brain, but at a low level of significance $(P<0.01$ without FWE). Figure 5 shows the $3 \mathrm{D}$ cortical representation of these brain regions. The results did not survive higher significance criteria; hence, these large clusters could not be reduced. However, we observed a trend of decreases in the frontal, temporal and parietal areas associated with suicide completion. The 10 most significant regions of decreased rCBF in terms of cluster size found to be most predictive of the suicide between these clinical profiles are listed in Table 4.

\section{Discussion}

The use of neuroimaging tools can be valuable in elucidating the neurobiological correlates underlying suicidal behavior. Here, we report the results of 21 completed suicides along with their comorbidities in an effort to better understand the alterations in metabolism and activity patterns that underlie this growing epidemic. We compared these subjects to a previously acquired database of SPECT scans from healthy patients, who have no prior psychiatric history and no family history of psychiatric behavior. This database allowed us to age and gender match this group to our suicide cohort. We also had extensive psychiatric profiles on each patient, allowing for deeper examination into the relationship between the SPECT findings and their clinical correlates.

Our primary finding demonstrates global decreases in blood flow and activity patterns in the suicide group versus a healthy comparison group. This finding was robust, even at $P<1 \times 10^{-10}$, and given the comorbidity of depression, ADHD, head trauma and bipolar diagnosis in our suicide cohort may indicate compromise among numerous neuronal pathways. However, although such comorbidity is often observable in clinical populations, not all of these cases end 
in suicide, thus differentiating those at risk is of value. The use of factorial modeling allowed us to differentiate distinct areas of neuronal deficits from a global pattern of neurological compromise, which were predictive of later suicide.

Over $90 \%$ of all suicides can be attributed to a psychiatric diagnosis, ${ }^{13}$ with depression and bipolar disorder carrying the highest risk. ${ }^{14,15}$ This was consistent within our cohort, all of whom were diagnosed as clinically depressed or as having a mood disorder through the BDI, Structured Clinical Interview

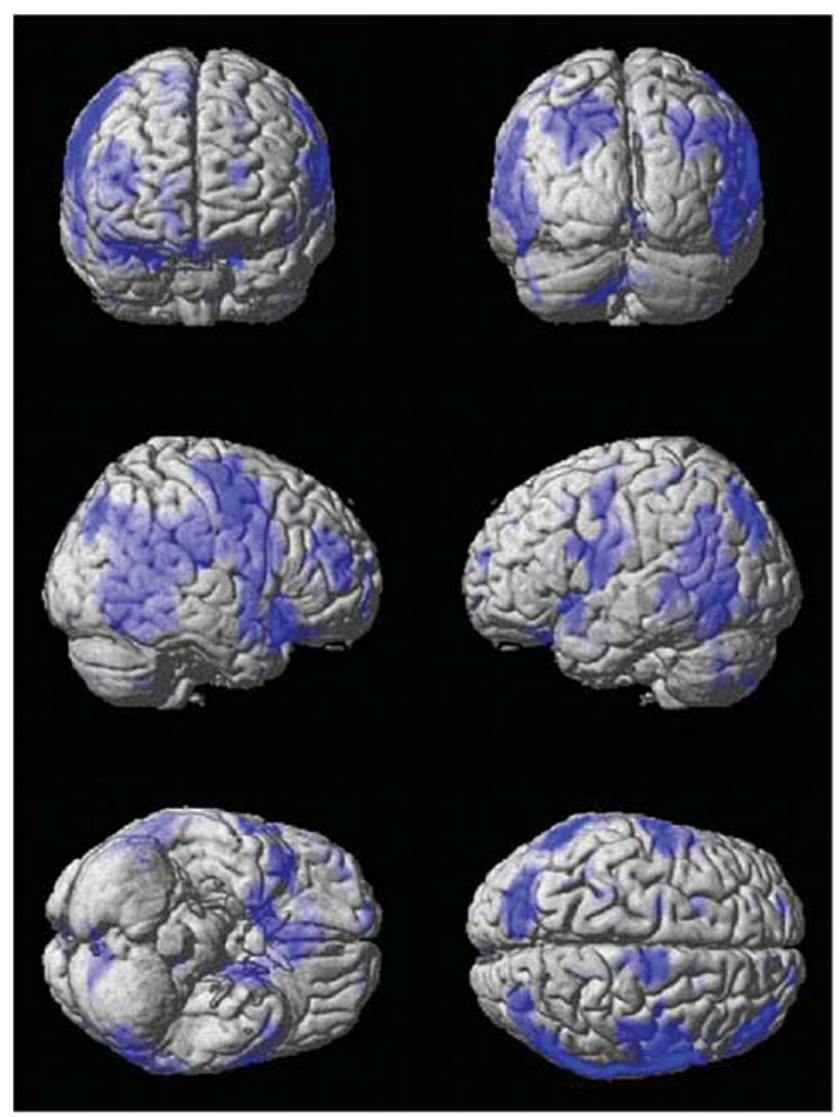

Figure 5 Three-dimensional (3D) surface images showing regional cerebral blood flow ( $\mathrm{rCBF}$ ) deficits predictive of suicide completion in suicide cohort versus a depressed non-suicidal clinical population. Cortical surface views showing areas of decreased rCBF predictive of suicide in completers versus depressed non-suicidal subjects with matched diagnostic profiles at $P<0.01$, without family-wise error (FWE). Significant decreases are in blue. for Diagnosis for DSM-IV or psychiatric evaluation. Neuroimaging has been a useful tool to demonstrate the link between depression, serotonergic dysfunction and suicide. Post-mortem studies of suicide patients found decreased 5-hydroxytryptamine $\left(5-\mathrm{HT}_{1 \mathrm{~A}}\right) / 5-\mathrm{HT}_{2 \mathrm{~A}}$ receptor binding in the ventrolateral prefrontal cortex, spurring in vivo imaging work in this area. ${ }^{16}$ SPECT imaging studies have confirmed reduced binding of $5-\mathrm{HT}_{2 \mathrm{Aa}}$ receptors in the frontal lobes of suicide attempters $^{17,18}$ and decreased serotonin transporter availability in suicide attempters with 5 -HTT polymorphisms ${ }^{19}$ indicative of serotonin abnormalities playing a role in suicide risk. Verbal fluency tasks have been used as a measure of executive ability in SPECT imaging, indicating prefrontal cortex function. ${ }^{20}$ Audenaert et al. ${ }^{21}$ showed decreased prefrontal perfusion correlated with poor performance on a verbal fluency task in depressed suicidal patients. Given that our study demonstrates decreased perfusion bilaterally in the superior/medial prefrontal cortex in a cohort of completed suicides with depression as a comorbid factor, it indicates potential deficits in the serotonergic system as well as in tasks associated with this brain region, including attention, memory and executive function.

In addition to elucidating the involvement of the serotonin system in suicidal behavior $\left(5-\mathrm{HT}_{1 \mathrm{~A}} / 5-\mathrm{HT}_{2 \mathrm{~A}}\right.$ receptor binding, 5-HTP receptor density, serotonin transporter polymorphisms), understanding the personality traits (impulsivity, aggression, anger, hopelessness) associated with suicidal ideation and suicide attempt is prudent. Our data demonstrate extreme deficits in the frontal cortex (the second and third most significant brain region decreased), indicating that our patient population was at high risk for problems with impaired judgment. Jollant et al. ${ }^{22}$ and others have shown that dysfunction in the orbitofrontal cortex is associated with suicide attempts and the tendency to make risky (disadvantageous) behavior choices. Within our cohort, not only were 13 subjects clinically diagnosed with attentional problems, 10 had a history of prior suicide attempt. High impulsivity is a predictor of self-aggression, ${ }^{23}$ and when combined with a prior history of suicide attempt, it amplifies the risk for suicide completion.

In examining our data, we wanted to further elucidate the relationship between the various hypoperfused brain regions and their respective influence on the self and the ability to cope with physical, mental and emotional pain. We found that the most pronounced region of decreased rCBF was in

Table 4 Top 10 regions of decreased rCBF predictive of suicide in depressed suicidal patients versus depressed non-suicidal patients

\begin{tabular}{|c|c|c|c|c|c|}
\hline Brain area & Cluster size & $t$ & $\boldsymbol{x}$ & $y$ & $z$ \\
\hline Temporal inferior lobe/right precentral gyrus & 4110 & 3.42 & 55 & -54 & -4 \\
\hline Left temporal lobe & 1422 & 3.17 & -58 & -58 & -10 \\
\hline Right temporal pole/left hippocampus/right rectus & 2206 & 3.15 & 46 & 20 & -16 \\
\hline Right superior motor area/cingulum & 1365 & 2.95 & 10 & -10 & 60 \\
\hline Right midfrontal lobe/frontal inferior triangularis & 406 & 2.95 & 42 & 52 & 16 \\
\hline Left superior parietal lobe & 969 & 2.93 & -18 & 78 & 50 \\
\hline Pre- and postcentral gyrus/left frontal inferior operculum & 774 & 2.85 & -56 & -2 & 50 \\
\hline Left superior temporal pole & 279 & 2.84 & -46 & 14 & -20 \\
\hline Left and right cerebellum & 190 & 2.81 & -8 & -74 & -50 \\
\hline Frontal medial orbital/right frontal superior & 106 & 2.77 & 12 & 64 & -12 \\
\hline
\end{tabular}

Abbreviation: rCBF, regional cerebral blood flow. 
the precuneus, an area important in episodic memory ${ }^{24}$ and reflections upon the self. ${ }^{25}$ This was followed by the prefrontal cortex, which not only was important in drive and executive function, but also in processing information about the self, ${ }^{25-27}$ as well as in coping with negative emotions ${ }^{28}$ and rejection. ${ }^{29}$ Other deficits include the rolandic operculum and postcentral gyrus, areas involved in processing sensory input, along with the caudate and thalamus, regions which contribute to emotional processing. Finally, we observed decreases in the insular cortex, a component of the limbic system involved in the management of physical and emotional pain. ${ }^{30}$ Overactivity of the right anterior insular cortex and right ventral prefrontal cortex have been found to be involved in processing frustration. ${ }^{31}$ Taken together, global deficits across these emotion encoding circuits may result in the underlying inability to properly perceive the self and to cope with life events, which result in the hopelessness and social isolation so commonly observed in those who commit suicide. This situation becomes exacerbated when combined with other factors, including depression, anxiety, post-traumatic stress disorder, drug use, head trauma and sexual abuse.

Developing new diagnostic markers is one way to reduce the prevalence of suicide. In our cohort of completed suicides, the subgenual cingulate cortex (BA 25) was found to be hypoperfused in 18 patients as compared with a healthy brain group. This is a significant finding, which implies that this region may serve as a potential neuroimaging biomarker for suicide. BA 25 is a region found to modulate human mood states and helps to integrate cognitive and emotional information. It is also an area well populated with serotonin receptors. If deficits in BA 25 are combined with decreased rCBF in the prefrontal cortex, an area associated with executive function and impulsivity, the risk factors for suicide increase exponentially. We believe this to be the case in our cohort, as 13 subjects were diagnosed with ADHD. Therefore, the combination of impulsivity, decreased rCBF in BA 25 and depression elevates patients into a high-risk category for suicidal behavior.

Our secondary finding demonstrates more subtle, global decreases in blood flow and activity patterns in the suicide group versus a matched clinical population of depressed, nonsuicidal individuals. Factorial modeling revealed this effect was weak (at $P<0.01$ without FWE). This suggests that the rCBF profile is too diffuse to distinguish specific patterns that will help the clinician identify the depressed, suicidal patient from the depressed non-suicidal patient. However, given the significant rCBF deficits between suicide completers and nonsuicidal depressed patients, it may be that the differentiating factor is the degree to which the metabolic activity of the brain is compromised versus specific regional differences in blood flow. A more definitive answer to this question will require increasing the number of patients studied to identify regional patterns between these two clinical populations.

The data in this paper not only support our previous work, ${ }^{9}$ but extend it to include significant deficits in rCBF to the emotional regulating circuits of the putamen, caudate, thalamus and insula, and also to highlight BA 25 as a potential biomarker for suicide. We propose that hypoperfusion of the prefrontal cortex and BA 25, along with dysregulation of the emotional circuits representing the self and the ability to cope with pain, may make a depressed patient more vulnerable to suicide. More research is needed to replicate our findings and to define the relative importance of any functional brain deficiencies predictive of suicide.

\section{Conflict of interest}

The authors declare no conflict of interest.

Acknowledgements. We thank David Keator for his consultation. Dr Daniel G Amen, MD, had full access to all the data in the study and takes responsibility for the integrity of the data and the accuracy of the data analysis.

Author contributions. Primary investigator: DGA; data analysis: DT; project management: KW.

1. Suicide Prevention (SUPRE). Geneva, Switzerland: World Health Organization. Available at: http://www.who.int/mental health/prevention/suicide/suicideprevent/en/.

2. Centers for Disease Control and Prevention NCfIPaC. Web-based Injury Statistics Query and Reporting System (WISQUARS). Available at: http://www.cdc.gov/injury/wisqars/ LeadingCauses.html (assessed via National Vital Statistics System 2007).

3. Nock MK, Hwang I, Sampson NA, Kessler RC. Mental disorders, comorbidity and suicidal behavior: results from the National Comorbidity Survey Replication. Mol Psychiatry 2010; 15: 868-876.

4. Bolton JM, Robinson J. Population-attributable fractions of axis I and axis II mental disorders for suicide attempts: findings from a representative sample of the adult, noninstitutionalized US population. Am J Public Health 2010; 100: 2473-2480.

5. Drevets WC, Price JL, Simpson Jr JR, Todd RD, Reich T, Vannier M et al. Subgenual prefrontal cortex abnormalities in mood disorders. Nature 1997; 386: 824-827.

6. Hirayasu Y, Shenton ME, Salisbury DF, Kwon JS, Wible CG, Fischer IA et al. Subgenual cingulate cortex volume in first-episode psychosis. Am J Psychiatry 1999; 156: 1091-1093.

7. Strakowski SM, Delbello MP, Adler CM. The functional neuroanatomy of bipolar disorder: a review of neuroimaging findings. Mol Psychiatry 2005; 10: 105-116.

8. Yucel K, McKinnon M, Chahal R, Taylor V, Macdonald K, Joffe R et al. Increased subgenual prefrontal cortex size in remitted patients with major depressive disorder. Psychiatry Res 2009; 173: 71-76.

9. Amen DG, Prunella JR, Fallon JH, Amen B, Hanks C. A comparative analysis of completed suicide using high resolution brain SPECT imaging. J Neuropsychiatry Clin Neurosci Fall 2009; $21:$ 430-439.

10. Brockmann $H$, Zobel A, Joe A, Biermann $K$, Scheef $L$, Schuhmacher $A$ et al. The value of HMPAO SPECT in predicting treatment response to citalopram in patients with major depression. Psychiatry Res 2009; 173: 107-112.

11. Friston KJ, Holmes AP, Worsley KJ, Poline JP, Frith CD, Frackowiak RSJ. Statistical parametric maps in functional imaging: a general linear approach. Hum Brain Mapp 1995; 2: $189-210$

12. Ashburner J, Friston KJ. Nonlinear spatial normalization using basis functions. Hum Brain Mapp 1999; 7: 254-266.

13. Mann JJ. Neurobiology of suicidal behaviour. Nat Rev Neurosci 2003; 4: 819-828.

14. Sun WJ, Xu L, Chan WM, Lam TH, Schooling CM. Depressive symptoms and suicide in 56000 older Chinese: a Hong Kong cohort study. Soc Psychiatry Psychiatr Epidemiol 2011.

15. Azorin JM, Kaladjian A, Besnier N, Adida M, Hantouche E, Lancrenon S et al. Suicidal behaviour in a French Cohort of major depressive patients: characteristics of attempters and nonattempters. J Affect Disord 2010; 123: 87-94.

16. Arango V, Underwood MD, Mann JJ. Postmortem findings in suicide victims. Implications for in vivo imaging studies. Ann NY Acad Sci 1997; 836: 269-287.

17. Audenaert K, Van Laere K, Dumont F, Slegers G, Mertens J, van Heeringen $C$ et al. Decreased frontal serotonin $5-\mathrm{HT} 2 \mathrm{a}$ receptor binding index in deliberate self-harm patients. Eur J Nucl Med 2001; 28: 175-182.

18. van Heeringen C, Audenaert K, Van Laere K, Dumont F, Slegers G, Mertens J et al. Prefrontal 5-HT2a receptor binding index, hopelessness and personality characteristics in attempted suicide. J Affect Disord 2003; 74: 149-158.

19. Bah J, Lindstrom M, Westberg L, Mannerås L, Ryding E, Henningsson S et al. Serotonin transporter gene polymorphisms: effect on serotonin transporter availability in the brain of suicide attempters. Psychiatry Res 2008; 162: 221-229.

20. Audenaert K, Brans B, Van Laere K, Lahorte P, Versijpt J, van Heeringen $\mathrm{K}$ et al. Verbal fluency as a prefrontal activation probe: a validation study using $99 \mathrm{~m} \mathrm{Tc-ECD}$ brain SPET. Eur J Nucl Med 2000; 27: 1800-1808.

21. Audenaert K, Goethals I, Van Laere K, Lahorte P, Brans B, Versijpt J et al. SPECT neuropsychological activation procedure with the verbal fluency test in attempted suicide patients. Nucl Med Commun 2002; 23: 907-916. 
22. Jollant F, Lawrence NS, Olie E, O'Daly O, Malafosse A, Courtet P et al. Decreased activation of lateral orbitofrontal cortex during risky choices under uncertainty is associated with disadvantageous decision-making and suicidal behavior. Neurolmage 2010; 51: 1275-1281.

23. Giegling I, Olgiati P, Hartmann AM, Calati R, Möller HJ, Rujescu D et al. Personality and attempted suicide. Analysis of anger, aggression and impulsivity. J Psychiatr Res 2009; 43: 1262-1271.

24. Lundstrom BN, Ingvar M, Petersson KM. The role of precuneus and left inferior frontal cortex during source memory episodic retrieval. Neurolmage 2005; 27: 824-834.

25. Feyers D, Collette F, D'Argembeau A, Majerus S, Salmon E. Neural networks involved in self-judgement in young and elderly adults. Neurolmage 2010; 53: 341-347.

26. Macrae CN, Moran JM, Heatherton TF, Banfield JF, Kelley WM. Medial prefrontal activity predicts memory for self. Cereb Cortex 2004; 14: 647-654.

27. Northoff G, Heinzel A, de Greck M, Bermpohl F, Dobrowolny H, Panksepp Self-referential processing in our brain-a meta-analysis of imaging studies on the self. Neurolmage 2006; 31: 440-457.
28. Davidson RJ, Irwin W. The functional neuroanatomy of emotion and affective style. Trends Cogn Sci 1999; 3: 11-21.

29. Eisenberger NI, Lieberman MD, Williams KD. Does rejection hurt? An FMRI study of social exclusion. Science 2003; 302: 290-292.

30. Singer T, Seymour B, O'Doherty J, Kaube H, Dolan RJ, Frith CD. Empathy for pain involves the affective but not sensory components of pain. Science 2004; 303: 1157-1162.

31. Abler B, Walter H, Erk S. Neural correlates of frustration. NeuroReport 2005; 16 $669-672$.

(c) Translational Psychiatry is an open-access journal SOMERTHHISRESERVED published by Nature Publishing Group. This work is licensed under the Creative Commons Attribution-Noncommercial-No Derivative Works 3.0 Unported License. To view a copy of this license, visit http://creativecommons.org/licenses/by-nc-nd/3.0/ 